\title{
Research on Optimal Control for the Vehicle Suspension Based on the Simulated Annealing Algorithm
}

\author{
Jie Meng, ${ }^{1}$ Qingzhang Chen, ${ }^{1}$ and Ren $\mathrm{He}^{2}$ \\ ${ }^{1}$ Department of Automotive Engineering, Changshu Institute of Technology, Changshu 215500, China \\ ${ }^{2}$ School of Automotive and Traffic Engineering, Jiangsu University, Zhenjiang 212013, China \\ Correspondence should be addressed to Qingzhang Chen; chenqz1973@sina.com
}

Received 17 February 2014; Accepted 1 March 2014; Published 3 April 2014

Academic Editor: Hamid R. Karimi

Copyright (c) 2014 Jie Meng et al. This is an open access article distributed under the Creative Commons Attribution License, which permits unrestricted use, distribution, and reproduction in any medium, provided the original work is properly cited.

\begin{abstract}
A method is designed to optimize the weight matrix of the LQR controller by using the simulated annealing algorithm. This method utilizes the random searching characteristics of the algorithm to optimize the weight matrices with the target function of suspension performance indexes. This method improves the design efficiency and control performance of the LQR control, and solves the problem of the LQR controller when defining the weight matrices. And a simulation is provided for vehicle active chassis control. The result shows that the active suspension using LQR optimized by the genetic algorithm compared to the chassis controlled by the normal LQR and the passive one, shows better performance. Meanwhile, the problem of defining the weight matrices is greatly solved.
\end{abstract}

\section{Introduction}

The suspension system is an important component of the vehicle, and its performance is a major factor that affects the vehicle ride comfort, operation, and stability. The traditional passive suspension is generally composed of the elastic component and damping components with the fixed parameters. And it is generally designed to meet a particular road, so it limits the vehicle performance. Therefore, industrial countries have already begun to study the active suspension and the semiactive suspension systems based on the active vibration control since the 1970s. In recent years, with the rapid development of the electronic technology, testing techniques, and system dynamics theories, the vibration isolation for the vehicle suspension system has changed from the traditional passive to the active vibration control. There is a lot of research in the suspension control areas in China. The application of the modern control theory in the vehicle suspension control also has a variety of algorithms, such as the PID, skyhook, optimal, and sliding-mode controllers [15].

The conventional fuzzy control lacks the control precision, and it is difficult to establish fuzzy rules. And the high complexity of the neural network, as a representative of the intelligent control technology, makes it impossible to analyze the various performance indexes accurately. Therefore, the system versatility cannot be satisfied.

Among the modern control theories, the optimal control is widely used, and its theoretical foundation is also perfect. As a kind of optimal control, the linear quadratic regulator (LQR) can provide the required optimal performance indexes, by determining the weighting matrix of the system state and control variables. And it can also give sufficient design space for designers. When applied to the vehicle active suspension design, the LQR control can put forward different objective functions for the different performance need.

That is to say, the LQR control can improve the vehicle performance by the comprehensive consideration of the various factors in the vehicle suspension system.

As to the LQR control, the choice of the weight matrix for the performance indexes has great influence on the control system performance. Usually, the weight matrix is determined manually according to the system's physical process, so it needs full understanding and sufficient experience of the system. Therefore, such "optimal" results are completely subjective and artificial. If the weight matrix is improperly 


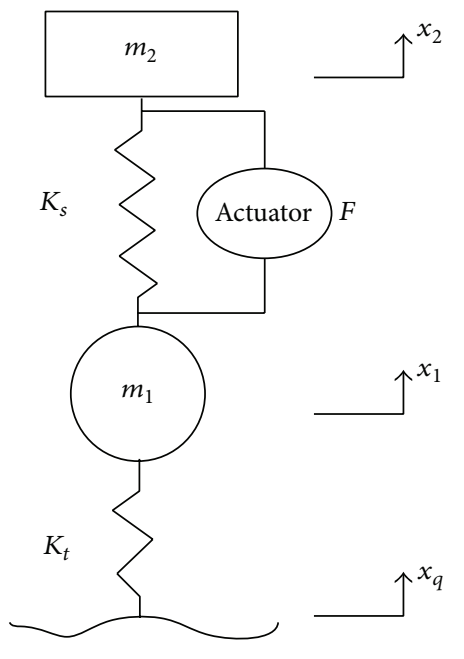

Figure 1: The quarter-vehicle body model of 2 DOF.

selected, the optimal solution of no practical significance. In addition, the choice of the weighted matrix relies too much on the designer's experience. The designers must adjust the weighted matrix step by step according to the system output until a satisfactory response value of output is acquired. Thus, the obtaining of feedback coefficient for the optimal control cannot guarantee the system to achieve the optimal aims [68].

At present, some random search methods such as the Genetic Algorithm (GA) and the Simulated Annealing (SA) have already caused the interest of many scholars. And they are already applied in the active suspension control field successfully. The simulated annealing algorithm is such a kind of the random search methods, and it is an approximation algorithm suitable and effective for solutions to the optimization problems in the mass general combinatorial fields. Compared with the previous approximate algorithm, the SA has the advantages of simple description, flexibility in use, universality, high efficiency, little constraint by the initial conditions, and so forth.

Due to the problems of the LQR in determining the weight matrix, a vehicle active suspension is selected as the control object, and the SA is applied in the LQR controller design optimized by the objective function of the active suspension performance index, aiming at improving the design efficiency and property.

\section{Establishment of the Active Suspension Control System}

For research convenience, a simplified quarter-vehicle model is selected as the research object, as Figure 1 shows.

In Figure 1, the symbol $m_{1}$ is the nonsprung mass and $m_{2}$ is the sprung mass. $K_{t}$ means the tire stiffness, and $K_{s}$ means the suspension stiffness. $F$ is the active force, and $X_{q}$ is the road input. $X_{1}$ is the displacement of the nonsprung mass and $X_{2}$ is the displacement of the sprung mass. The dynamics equation can be got from Figure 1:

$$
\begin{aligned}
& m_{2} \ddot{x}_{2}=U-K_{s}\left(x_{2}-x_{1}\right), \\
& m_{1} \ddot{x}_{1}=-U+K_{s}\left(x_{2}-x_{1}\right)-K_{t}\left(x_{1}-x_{q}\right) .
\end{aligned}
$$

The state variables and the output variables are selected, respectively, as follows:

$$
\begin{aligned}
& X=\left(\dot{x}_{2}, \dot{x}_{1}, x_{2}, x_{1}, x_{q}\right)^{T}, \\
& Y=\left(\ddot{x}_{2}, x_{2}-x_{1}, x_{1}-x_{q}\right)^{T} .
\end{aligned}
$$

The control input is the active force $F$. And the filtering white noise is used to simulate the real road input:

$$
\dot{x}_{q}(t)=-2 \pi f_{0} x_{q}(t)+2 \pi \sqrt{G_{0} V} \omega(t) .
$$

In the formula, the symbol $G_{0}$ represents the pavement roughness coefficient $\left(\mathrm{m}^{3} /\right.$ cycle). The symbol $V$ means the vehicle speed. The $\omega(t)$ means the Gaussian white noise with zero mathematical expectations. The $f_{0}$ is the lower cutoff frequency.

Then, the system state equation can be achieved:

$$
\begin{aligned}
& \dot{\mathbf{x}}=\mathbf{A x}+\mathbf{B U} \\
& \mathbf{y}=\mathbf{C} \mathbf{x}+\mathbf{D U}
\end{aligned}
$$

Annotation. Consider the following:

$$
\mathbf{A}=\left[\begin{array}{ccccc}
0 & 0 & -\frac{K_{s}}{m_{2}} & \frac{K_{s}}{m_{2}} & 0 \\
0 & 0 & \frac{K_{s}}{m_{1}} & -\frac{K_{t}+K_{s}}{m_{1}} & \frac{K_{t}}{m_{1}} \\
1 & 0 & 0 & 0 & 0 \\
0 & 1 & 0 & 0 & 0 \\
0 & 0 & 0 & 0 & -2 \pi f_{0}
\end{array}\right],
$$$$
\mathbf{B}=\left[\begin{array}{cc}
0 & \frac{1}{m_{2}} \\
0 & \frac{-1}{m_{1}} \\
0 & 0 \\
0 & 0 \\
2 \pi \sqrt{G_{0} v} & 0
\end{array}\right]
$$

$$
\begin{gathered}
\mathbf{C}=\left[\begin{array}{ccccc}
0 & 0 & -\frac{K_{s}}{m_{2}} & \frac{K_{s}}{m_{2}} & 0 \\
0 & 0 & 1 & -1 & 0 \\
0 & 0 & 0 & 1 & -1
\end{array}\right], \quad \mathbf{D}=\left[\begin{array}{ll}
0 & 0 \\
0 & 0 \\
0 & 0
\end{array}\right], \\
\mathbf{U}=\left[\begin{array}{c}
\omega(t) \\
F
\end{array}\right] .
\end{gathered}
$$




\section{Optimal Controller Design for Active Suspension}

The main performance for automobile suspension design includes three indexes: the body vertical vibration acceleration which represents the car ride, the suspension dynamic travel which represents the body posture and the suspension structure, and the dynamic load of the tyre which represents the tire grounding characteristic.

Therefore, the target performance index $\mathbf{J}$ can be set as the mean integral value of the weighted square sum of the body acceleration, the suspension dynamic travel, and the tire's dynamic displacement:

$$
\mathbf{J}=\lim _{T \rightarrow \infty} \frac{1}{T} \int_{0}^{T}\left[q_{1}\left(x_{1}-x_{q}\right)^{2}+q_{2}\left(x_{2}-x_{1}\right)^{2}+q_{3} \ddot{x}_{2}^{2}\right] d t .
$$

In the equation, the symbols $q_{1}, q_{2}$, and $q_{3}$ represent the weight coefficients of the body acceleration, the suspension dynamic travel, and the tire's dynamic displacement, respectively. The choice of the three weight coefficients depends on the designer's tendency of the suspension performance.

Then, formula (4) is substituted into the performance index $\mathbf{J}$ and the following formula can be got:

$$
\mathbf{J}=\lim _{x \rightarrow \infty} \frac{1}{T} \int_{0}^{T}\left[\mathbf{x}^{\mathrm{T}} \mathbf{Q} \mathbf{x}+\mathbf{u}^{\mathrm{T}} \mathbf{R} \mathbf{u}+\mathbf{2} \mathbf{x}^{\mathrm{T}} \mathbf{N u}\right] d t .
$$

The symbols $\mathbf{Q}, \mathbf{R}$, and $\mathbf{N}$ are noted as follows:

$$
\begin{aligned}
& \mathbf{Q}=\left[\begin{array}{ccccc}
0 & 0 & 0 & 0 & 0 \\
0 & 0 & 0 & 0 & 0 \\
0 & 0 & q_{2}+\frac{K_{s}^{2}}{m_{2}^{2}} & -q_{2}-\frac{K_{s}^{2}}{m_{2}^{2}} & 0 \\
0 & 1 & -q_{2}-\frac{K_{s}^{2}}{m_{2}^{2}} & q_{1}+q_{2}+\frac{K_{s}^{2}}{m_{2}^{2}} & -q_{1} \\
0 & 0 & 0 & -q_{1} & q_{1}
\end{array}\right] \text {, } \\
& \mathbf{N}=\frac{1}{m_{2}^{2}}\left[\begin{array}{c}
0 \\
0 \\
-K_{s} \\
K_{s} \\
0
\end{array}\right] \text {. } \\
& \mathbf{R}=\frac{1}{m_{2}^{2}},
\end{aligned}
$$

The feedback matrix $K$ of the optimal control can be achieved from the Riccati matrix formula:

$$
\mathbf{P A}+\mathbf{A}^{\mathrm{T}} \mathbf{P}-(\mathbf{P B}+\mathbf{N}) \mathbf{R}^{-1}\left(\mathbf{B}^{\mathrm{T}} \mathbf{P}+\mathbf{N}^{\mathrm{T}}\right)+\mathbf{Q}=\mathbf{0} .
$$

According to the feedback variables $\mathbf{X}(\mathbf{t})$ at any time, the optimal control force $\mathbf{F}$ can be got by the following equation:

$$
\mathbf{F}=-\mathbf{K X}(\mathbf{t}) \text {. }
$$

The $\mathbf{K}$ value can be acquired through the design function for the optimal controller in Matlab:

$$
[\mathbf{K}, S, e]=\operatorname{LQR}(\mathbf{A}, \mathbf{B}, \mathbf{Q}, \mathbf{R}, \mathbf{N}) .
$$

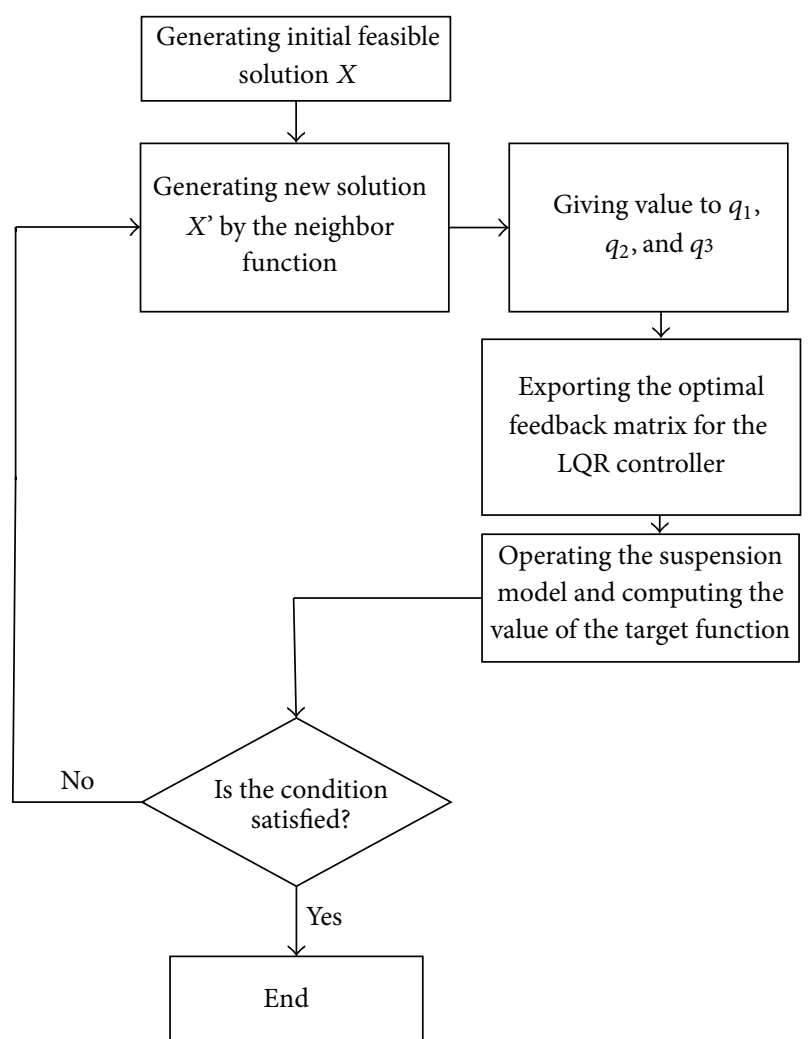

FIGURE 2: The optimization process for the LQR controller by the SA.

According to the above formulas, the optimal control design depends on the choice of weighting coefficients totally. Previous designs often rely on the designer's own experience through repeated trials. Though corresponding optimal results can be got, a large subjectivity is met.

Therefore, the simulated annealing algorithm is used to optimize the weights coefficient matrix in order to reduce the controller design time and avoid the subjectivity at the same time.

\section{Optimum Controller Design Based on the Simulated Annealing Algorithm}

The simulated annealing (SA) algorithm is put forward by Metropolis at the earliest. His starting point is based on the similarity of the solid material annealing process to the general combinatorial optimization problem. The SA algorithm is a general optimization algorithm with its annealing process composed of three processes: the heating process, the isothermal process, and the cooling process $[9,10]$.

The optimum controller design based on simulated annealing algorithm is a combination of the SA and LQR control method. The SA is used to optimize and solve the weight matrix, and the LQR is used to calculate the system performance index. 


\subsection{Design Model of the LQR Controller Optimized by the SA}

4.1.1. Designation of the Optimized Variable. The weights coefficient is taken as the optimized variable of the SA:

$$
X=\left[q_{1}, q_{2}, q_{3}\right]^{T} .
$$

4.1.2. Establishment of the Objective Function. Because there is difference in the order of magnitude and the units, the optimization aim of the objective function is taken as follows:

$$
\begin{gathered}
\text { Minimum } f(\mathbf{X})=\frac{\operatorname{RMS}\left[\ddot{x}_{3}(\mathbf{X})\right]}{\operatorname{RMS}\left[\ddot{x}_{3 \text { pass }}(\mathbf{X})\right]} \\
+\frac{\operatorname{RMS}\left[\left(x_{3}-x_{2}\right)(\mathbf{X})\right]}{\operatorname{RMS}\left[\left(x_{3}-x_{2}\right)_{\text {pass }}(\mathbf{X})\right]} \\
+\frac{\operatorname{RMS}\left[\left(x_{1}-x_{q}\right)(\mathbf{X})\right]}{\operatorname{RMS}\left[\left(x_{1}-x_{q}\right)_{\text {pass }}(\mathbf{X})\right]} \\
\frac{\operatorname{RMS}\left[\ddot{x}_{3}(\mathbf{X})\right]}{\operatorname{RMS}\left[\ddot{x}_{3 p a s s}(\mathbf{X})\right]}<1 \\
\frac{\operatorname{RMS}\left[\left(x_{3}-x_{2}\right)(\mathbf{X})\right]}{\operatorname{RMS}\left[\left(x_{3}-x_{2}\right)_{\text {pass }}(\mathbf{X})\right]}<1 \\
\frac{\operatorname{RMS}\left[\left(x_{1}-x_{q}\right)(\mathbf{X})\right]}{\operatorname{RMS}\left(x_{1}-x_{q}\right)_{\text {pass }}(\mathbf{X})}<1 \\
\quad 0.1<\mathbf{X}_{i}<10^{6}, \quad i=1,2,3 . \\
X=\left[q_{1}, q_{2}, q_{3}\right], \quad, \quad
\end{gathered}
$$

In the equations, the symbols are noted as follows:

[RMS]: the root mean square value of relevant data; $\ddot{x}_{3 \text { pass }}:$ the vertical acceleration of the passive suspension;

$\left(x_{3}-x_{2}\right)_{\text {pass }}$ : the dynamic displacement of the suspension;

$\left(x_{1}-x_{q}\right)_{\text {pass }}$ : the dynamic displacement of the tyre;

$X$ : the coefficient matrix of the LQR.

4.2. The Optimization Process of the SA. The optimization process of the SA is shown in Figure 2.

(1) Creation of the initial feasible solution: $X=$ $\left[q_{1}, q_{2}, q_{3}\right]$. The initial temperature $t_{0}$ is determined, and random initial solution is selected together with the iterations times at any time which is the Metropolis chain length.

(2) Then, new solutions are created through the neighborhood function according to the current solution $X$. This process continues until the new solutions are feasible, and then their optimization aims and comprehensive aims are evaluated.

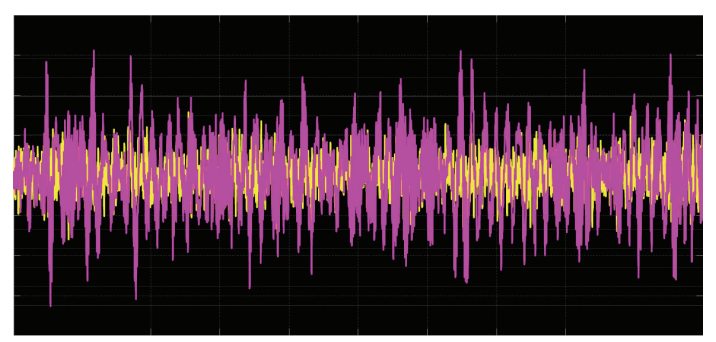

FIGURE 3: Vertical acceleration of the active and passive suspension.

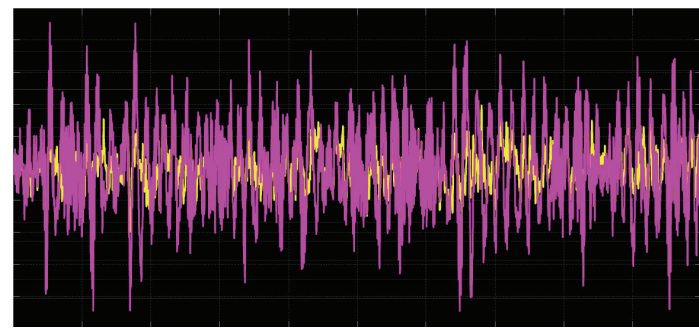

FIgURE 4: Dynamic travel of the active and passive suspension.

(3) Make sure that the criteria are met at current temperature. If ok, then the process continues or returns to step (2).

(4) Then, the cooling process is operated and $t_{k+1}=$ $t_{k} \exp (-c k)$. The $c$ is 0.9 .

(5) Make sure the algorithm meets the principle of termination. If it is ok, then the process is terminated or returns to step (2) again.

\section{Simulation and the Results}

Under the environment of the Matlab/Simulink, simulations are performed as to the active suspensions controlled by the LQR controller optimized by the SA and other controlling manners. The B-grade road in the standard fileISO/TC108/SC2N67 is selected according to the previous model. A random road input is generated at the speed of $20 \mathrm{~km} / \mathrm{h}$, and $G_{0}$ is $5 * 10^{-6} \mathrm{~m}^{3} /$ cycle. And $m_{1}$ is $50 \mathrm{~kg}, m_{2}$ is $300 \mathrm{~kg}, m_{3}$ is $50 \mathrm{~kg}, k_{1}$ is $200000 \mathrm{~N} / \mathrm{m}$, and $k_{2}$ is $20000 \mathrm{~N} / \mathrm{m}$. The system simulation results are shown in Figures 3 to 5.

The LQR weights that the matrix gets from the simulation are [118.39 49.74 61.42].

In order to validate the superiority of the SALQR in the suspension control, the performance results of the suspension in Simulink simulation are compared and are shown in Table 1 .

It can be seen from Figures 3, 4, and 5 and Table 1 the following.

(1) The SALQR controller significantly reduces the vertical acceleration of the suspension. In this regard, its effect is very well. 
TABLE 1: Performance contrast of the suspension of different control measures.

\begin{tabular}{lccc}
\hline Performance indexes & Unit & Passive suspension & $\begin{array}{c}\text { The mean square root value } \\
\text { Conventional LQR }\end{array}$ \\
\hline Vertical acceleration of suspension & $\mathrm{m} / \mathrm{s}^{2}$ & 2.8358 & 1.6342 \\
Dynamic travel of suspension & $\mathrm{mm}$ & 40.7465 & 47.7899 \\
Dynamic deflection of the tyre & $\mathrm{mm}$ & 11.8446 & 6.5684 \\
\hline
\end{tabular}

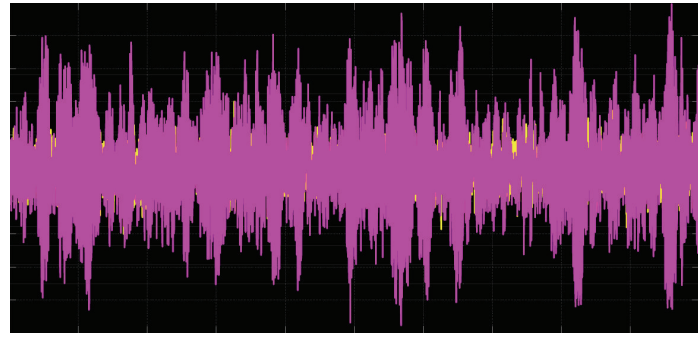

FIGURE 5: Dynamic deflection of the active and passive suspension.

(2) In regard of the suspension's dynamic travel, the SALQR also gives good effect compared to the passive suspension and the conventional LQR suspension.

(3) In the regard of the tire's dynamic deflection, the effect of the SALQR is no good compared to other controlling manners. But the weakness is not so significant to affect the whole performance of the suspension.

\section{Conclusions}

In view of the reliance on the designer's experience of the conventional LQR controller, a controller combining the SA and LQR is designed, and it has the following advantages.

(1) The dependence on the designer's experience is reduced and the design convenience and efficiency are improved.

(2) The controller performance is enhanced compared to the conventional LQR controller; therefore, the SA has a wide application prospect in the active control of automobile suspension.

\section{Conflict of Interests}

The authors declare that there is no conflict of interests regarding the publication of this paper.

\section{Acknowledgments}

The authors gratefully acknowledge the financial support from the National Natural Science Foundation of China (no. 51275060) and the Natural Science Foundation of Jiangsu Province (no. BK2011367).

\section{References}

[1] H. Zhang, J. Wang, and Y. Shi, "Robust $H_{\infty}$ sliding-mode control for Markovian jump systems subject to intermittent observations and partially known transition probabilities," Systems \& Control Letters, vol. 62, no. 12, pp. 1114-1124, 2013.

[2] Y. G. Zhao and J. Chen, "Co-simulation on semi-active suspension of vehicles," Noise and Vibration Control, vol. 31, no. 5, pp. 104-107, 2011 (Chinese).

[3] L. Chai, T. Sun, and J. Feng, "Design of the LQG controller for active suspension system based on analytic hierarchy process," Automotive Engineering, vol. 32, no. 8, pp. 712-718, 2010.

[4] H. Zhang, Y. Shi, and J. Wang, "Observer-based tracking controller design for networked predictive control systems with uncertain Markov delays," International Journal of Control, vol. 86, no. 10, pp. 1824-1836, 2013.

[5] R. Solea, A. Filipescu, and D. Cernega, "Lateral motion control of four-wheels steering vehicle using a sliding-mode controller," in Proceedings of the 29th Chinese Control Conference (CCC '10), pp. 3699-3703, Beijing, China, July 2010.

[6] L. Wang, The Intelligence Algorithm and Its Application, Tsinghua University press, Beijing, China, 2011.

[7] J. Wang, J. Zhang, and X. Wei, "Multi-objective optimization design of gear reducer based on simulated annealing algorithms," Transactions of the Chinese Society of Agricultural Machinery, vol. 37, no. 10, pp. 120-123, 2006.

[8] R. Zhou and Z. Guo, "Research on active suspension control technology," Journal of Tongji University, vol. 197, pp. 176-180, 2012.

[9] D. Liu, Y. Tang, and H. Y. Gu, "The development of automotive active suspension control system," Hydraulics Pneumatics \& Seals, vol. 5, no. 4, pp. 21-25, 2010.

[10] W. J. Yan, D. Dong, and W. R. Wang, "On fuzzy strategy of nonlinear semi-active suspension system," Control Engineering of China, vol. 18, no. 6, pp. 941-946, 2011. 


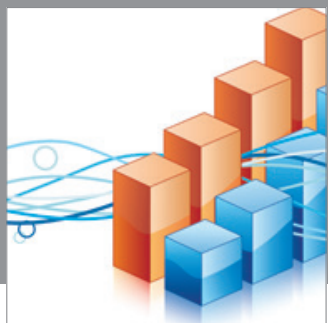

Advances in

Operations Research

mansans

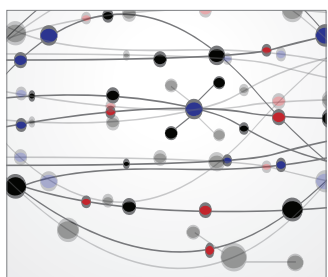

The Scientific World Journal
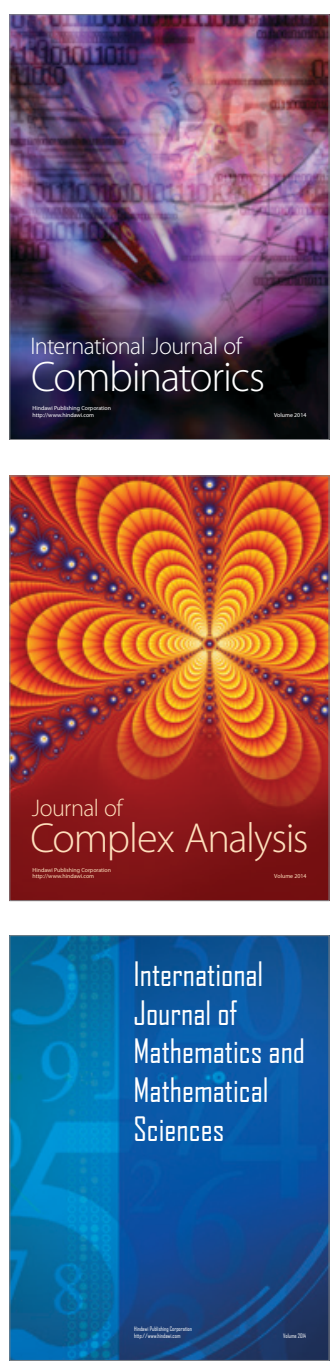
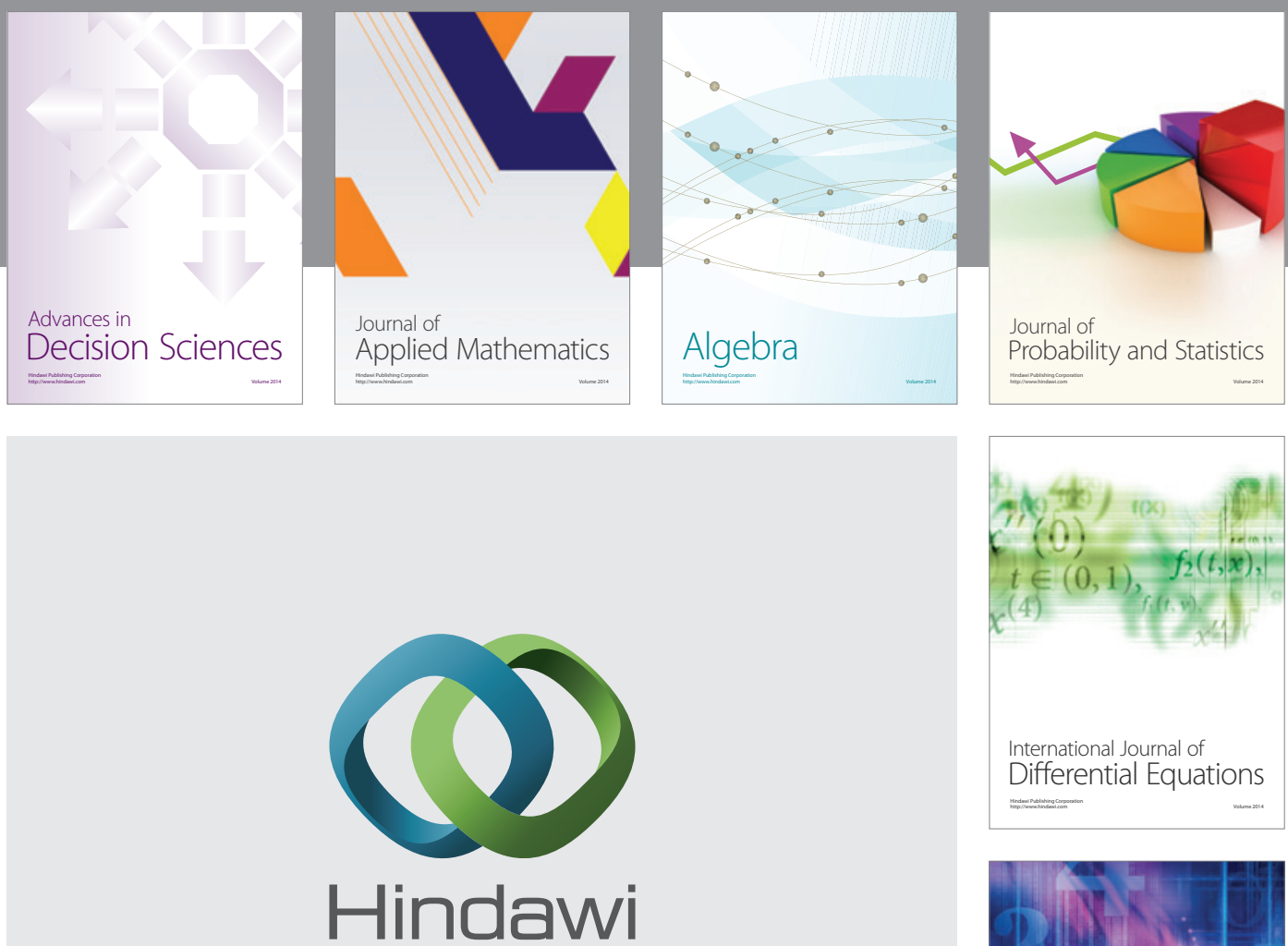

Submit your manuscripts at http://www.hindawi.com
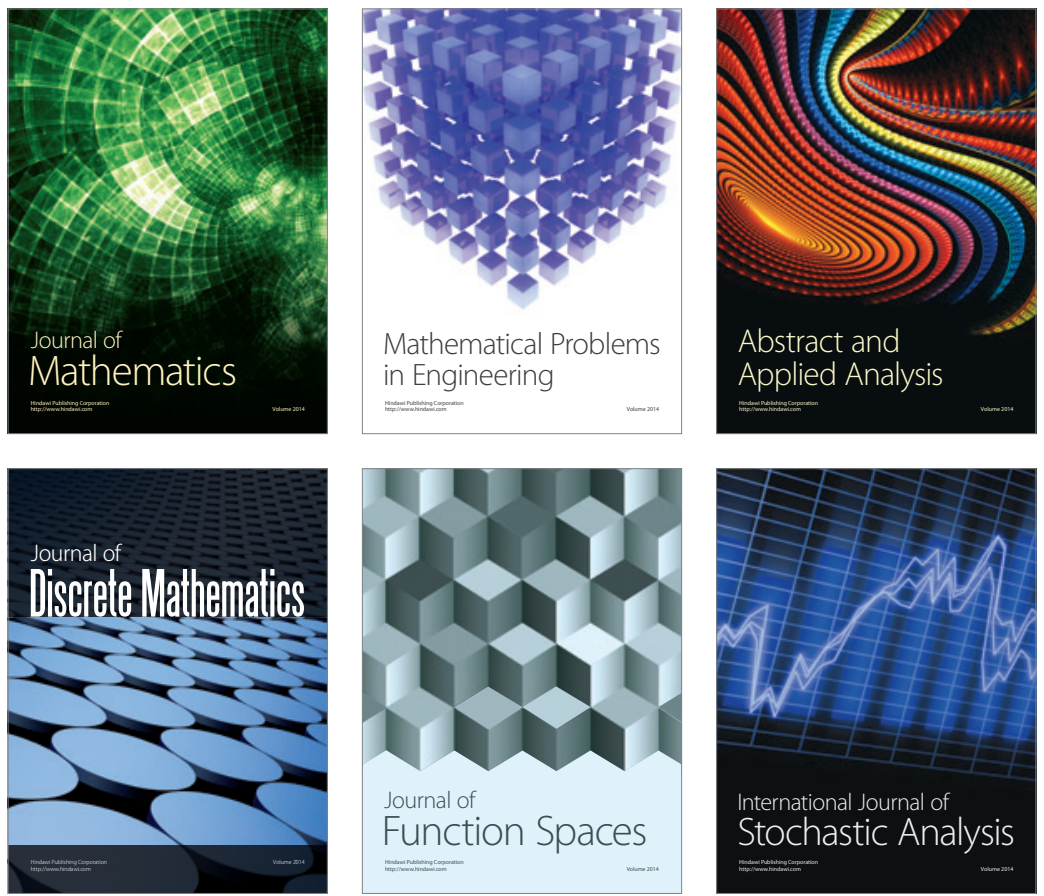

Journal of

Function Spaces

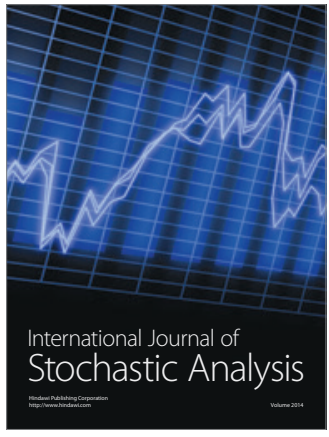

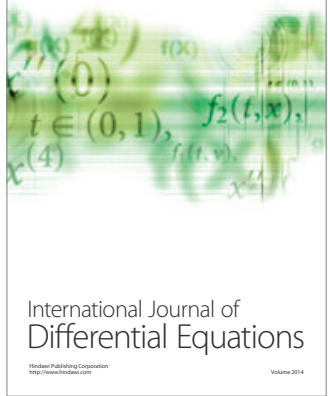
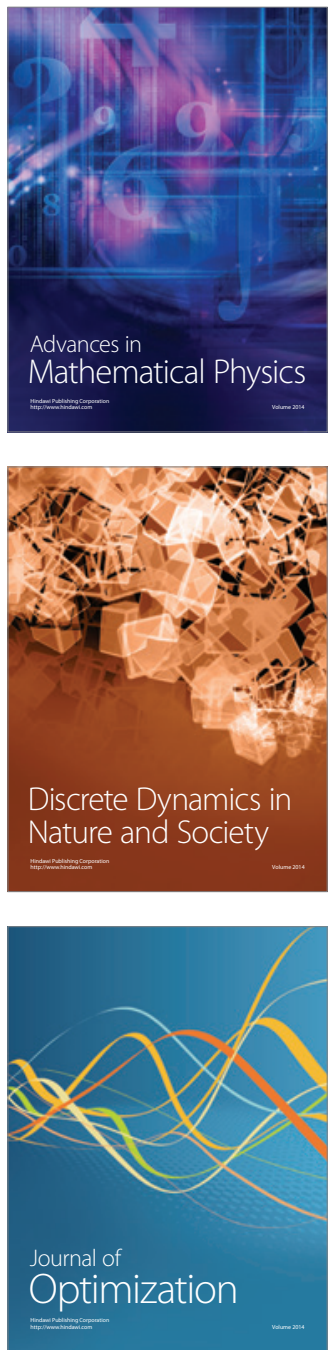